\title{
Le DES de réanimation : les vérités sont parfois bonnes à dire
}

\section{Critical care medicine as a primary speciality: facts and truths}

\author{
F. Fourrier $\cdot$ A. Vieillard-Baron $\cdot$ J. Chastre \\ C SRLF et Springer-Verlag France 2014
}

La demande de création d'un diplôme d'études spécialisées (DES) de réanimation, que les différentes composantes de notre spécialité soutiennent, s'inscrit dans le cadre de la réforme du troisième cycle et du postinternat. C'est dans ce cadre qu'il faut l'analyser. C'est une réforme de bon sens dont l'objectif essentiel est d'adapter la formation des médecins généralistes et des spécialistes aux besoins de santé publique du futur en assurant la maîtrise des flux et de la territorialité. Elle prône la disparition des diplômes d'études spécialisées complémentaires (DESC) qualifiants et l'organisation d'une formation professionnalisante plus précoce.

Quelle que soit la spécialité, il n'est plus logique qu'un étudiant terminant aujourd'hui son internat muni d'un diplôme de spécialiste ne soit pas considéré - et souvent ne se considère pas lui-même - comme apte à exercer son métier. La prolongation des études par l'acquisition de sur-sp écialités ou surtout pour bénéficier d'une formation pratique séniorisée conduit à allonger la durée de formation et à retarder le remplacement des générations de médecins. À la phase initiale des discussions avec les commissions ministérielles chargées de la réforme, beaucoup de spécialités ont d'abord proposé d'augmenter la durée des DES pour assurer cette séniorisation : l'anesthésie-réanimation à six ans, les spécialités médicales à cinq, etc. La tentation des enseignants est de toujours vouloir enseigner plus longtemps en pensant que la durée conditionne la qualité d'une formation. C'est faux, et heureusement la prolongation des cursus médicaux a été rapi-

\section{F. Fourrier $(\square)$}

Président du Collège National des Enseignants de Réanimation (CNER), département universitaire de réanimation - Université de Lille 2 - CHRU de Lille, service de réanimation polyvalente, hôpital Roger Salengro, F-59037 Lille cedex

e-mail : francois.fourrier@chru-lille.fr

A. Vieillard-Baron

Secrétaire général du CNER, CHU Ambroise Paré, service de réanimation médicale, F-92104 Boulogne-Billancourt

J. Chastre

Président de la sous-section 48/02 du CNU,

CHU la Pitié-Salpêtrière, service de réanimation médicale,

F-75651 Paris cedex 13 dement écartée. Aujourd'hui déjà un médecin spécialiste commence à peine d'exercer son métier à l'âge où la plupart des professionnels subissant une sélection de même niveau sont largement engagés dans une carrière valorisée. Les diplômés de l'ENA sont déjà sous-préfets ou conseillers d'État, les polytechniciens sont directeurs ou ingénieurs en chef, les diplômés des grandes écoles sont depuis longtemps pourvus de masters en administration du commerce et exercent des fonctions de manager, etc. Seuls les médecins attendent souvent l'âge de 32 à 34 ans dans de nombreuses spécialités pour débuter leur carrière professionnelle. Il faut s'interroger sur ce que cela représente aussi à titre individuel et familial. Gagner sa vie et être en responsabilité très tard n'est pas le meilleur service que nous rendons à nos étudiants. C'est coûteux, c'est inutile, car nous pouvons certainement mieux faire et plus vite, former les internes en intégrant la séniorisation dans leur cursus, en utilisant les moyens modernes d'enseignement, en adaptant nos modalités pédagogiques aux résultats des réformes des premier et deuxième cycles. Celles-ci vont apprendre aux futurs médecins à manier les bases de données, à décider dans les situations d'incertitude diagnostique, à partager les compétences, à s'intégrer dans les filières de soin. Le récent plaidoyer du président de l'ordre des médecins auprès du président de la République n'est pas si éloigné de ces objectifs quand il prône une meilleure intégration de la formation médicale à la société actuelle dans son ensemble. Oui, la réforme $\mathrm{du}$ troisième cycle et du postinternat possède une logique de santé publique et universitaire, et elle est souhaitable et urgente. Et la réanimation est une spécialité comme les autres qui ne peut échapper à cette orientation.

Dans le cadre de cette réforme, nous avons souhaité rejoindre le collectif des spécialités médicales «primaires » pour développer une formation à la réanimation par un DES axé sur l'acquisition d'une « culture médicale » et une séniorisation précoce. Encore une fois, la réanimation est une spécialité comme les autres et elle ne peut échapper aux mêmes contraintes. Et donc nous avons souhaité que cesse le gâchis par érosion d'un DESC qualifiant de trois ans dont la moitié des inscrits, même s'ils en apprécient probablement l'enseignement, disparaissent en cours de formation parce que celle-ci est trop longue et son accès difficile. 
Nos collègues d'anesthésie-réanimation (AR) s'opposent avec vigueur à la demande que nous avons présentée devant la commission de réforme du troisième cycle. Un éditorial récent publié sur le site de la Société française d'anesthésie réanimation (Sfar) en est la dernière illustration. Nous souhaitons y répondre.

Il est faux de dire que, comme l'AR, toutes les spécialités médicales s'opposent à notre demande. Il est faux de dire que la création d'un DES de réanimation menace ces spécialités d'une quelconque façon. Nous avons rencontré nos confrères des collèges et collégiales des spécialités médicales pour leur montrer que, comparé au DESC actuel, la création d'un DES de réanimation n'exercerait aucun impact supplémentaire sur la formation des internes de leur discipline. L'accès des internes de ces spécialités à nos services est garanti, leur formation sera assurée comme aujourd'hui. Le choix plus précoce qui s'imposera aux nouveaux internes ne conduira pas à plus de perte de ressources humaines spécifiques à ces disciplines. Au contraire, notre maquette prévoit d'orienter les internes qui choisiront la réanimation vers un cursus médical préférentiel dans une spécialité — ou plusieurs s'ils souhaitent une formation de type médecine interne. Ces internes inscrits au DES de réanimation suivront ainsi pour la moitié de leur internat un cursus de formation en stages de médecine propre à leur donner la culture médicale indispensable à l'exercice de la réanimation.

Ce point est crucial parce que $80 \%$ des patients admis dans les services de réanimation ne sont pas des patients chirurgicaux ou de type périopératoire. Ce sont des patients « médicaux », au sens où ils présentent des défaillances viscérales engageant leur pronostic vital, intégrées ou dépendantes d'une maladie de plus en plus souvent chronique et de plus en plus souvent multiviscérale et dont la prise en charge souvent complexe nécessite une formation appropriée dans les domaines concernés.

Il est faux de dire que le DES d'anesthésie-réanimation (DESAR) prépare correctement les internes à la prise en charge de ces patients. Les internes du DESAR sont d'abord formés à l'anesthésie et aux situations périopératoires - et c'est tout à fait normal et nécessaire. Ils ne suivent pendant leur cursus aucun stage en service de médecine et n'acquièrent que peu d'expérience clinique des patients médicaux. Ils ne les fréquentent qu'à travers les consultations préopératoires, au bloc ou en postopératoire. Ils ne les rencontrent souvent qu'en réanimation et dans des situations bien éloignées de leur maladie initiale ou chronique.

La position de nos collègues d'AR sur ce plan est évidemment opposée : ils considèrent, répètent et assument l'idée que le DESAR est parfaitement capable à lui seul d'assurer la formation initiale et le remplacement de tous les réanimateurs qui vont quitter la scène professionnelle.

En l'absence de toute donnée démographique précise, ils font le calcul qu'aujourd'hui $30 \%$ des anesthésistes-réanima- teurs exercent la réanimation. Ainsi, la filiarisation actuelle du DESAR permettrait sans coup férir d'assurer la formation de tous les réanimateurs français et même plus. C'est faux, le pourcentage de $30 \%$ de 9000 anesthésistes-réanimateurs est largement surestimé : il voudrait dire que près de 3000 anesthésistes-réanimateurs exercent actuellement dans les unités de réanimation. Ce chiffre amalgame les anesthésistes-réanimateurs en poste dans les réanimations à ceux qui participent parfois à la permanence des soins ; il assimile les DESC de réanimation actuellement en poste et dont la spécialité primaire était l'AR à des anesthésistes-réanimateurs « purs et durs », comme si par exemple nos collègues cardiologues ou pneumologues comptabilisaient les DESC en poste en réanimation et passés initialement par leurs spécialités comme appartenant à leur discipline. Il ne prend pas non plus en compte les très nombreux praticiens qui exercent aujourd'hui en réanimation sans diplôme, mais en vertu d'une expérience attestée et dont il sera nécessaire d'assurer le remplacement par des réanimateurs diplômés et qualifiés. Enfin, dans l'analyse démographique, il est fait complètement abstraction des besoins croissants de l'anesthésie en milieu libéral et par ailleurs de l'implication des anesthésistes-réanimateurs dans de multiples domaines : urgence, Samu, soins palliatifs, douleur.

Au-delà d'une querelle de chiffres qui nécessiterait que l'AR réalise une enquête précise de ses besoins dans la totalité des domaines qu'elle souhaite investir, il se pose ici un véritable choix de santé publique qui concerne toutes les spécialités et finalement toute la population. Est-il souhaitable ou non que l'AR soit la discipline unique qui forme tous les futurs réanimateurs? Si la réponse est oui, il faut accepter l'idée que la réanimation devienne un lieu où les dimensions multidisciplinaires et la culture médicale seront peu, voire plus représentées.

Nous pensons quant à nous que la réponse est non. Il est faux de dire que la formation unique à la réanimation par le DESAR satisfera les besoins quantitatifs du futur, il est faux de dire qu'elle pourra assurer la formation des médecins nécessaires à la prise en charge de malades médicaux complexes. Ce n'est pas seulement la très haute qualité des publications de recherche qui peut permettre d'y parvenir, même si la production scientifique d'une discipline est un critère auquel nous adhérons totalement et dont notre discipline peut s'enorgueillir au même titre que l'AR. C'est l'enseignement, la formation en stage, l'intégration dans un « bain de culture médicale » auprès de praticiens séniors habitués à cette dimension. Il est de plus parfaitement contradictoire de la part de nos collègues d'AR de soutenir que leur discipline est à même d'assurer la formation de tous les réanimateurs et en même temps de prôner une réanimation forte et multiculturelle. Comment pourrait-elle être forte lorsque la discipline d'AR devra assurer seule une formation théorique et pratique de grande qualité dans tous les domaines qu'elle souhaite couvrir ? Comment pourrait-elle être multiculturelle si cela 
conduit à ce que la seule discipline formatrice soit l'AR, tout en prônant qu'un schéma de formation commune permette à toutes les disciplines médicales et chirurgicales d'accéder à l'exercice de la réanimation? C'est contradictoire et excessif.

Il est faux de dire que la qualification de réanimateur n'intéresse pratiquement personne. C'est offensant pour les médecins qui en disposent ou ont fait souvent des sacrifices pour l'obtenir ; c'est déprécier le travail des commissions ordinales qui certifient à nos patients que les médecins qui les soignent en réanimation sont aptes à le faire ; c'est peu confraternel pour les médecins étrangers qui sont de plus en plus nombreux à en faire la demande et s'engagent pour ce faire dans une formation continue difficile. Le fait que nombre de réanimateurs n'aient pas demandé expressément leur changement de discipline ordinale, après l'obtention de leur spécialité primaire, tient d'abord à la complexité des procédures officielles et aux changements répétés de statut. On ne peut rayer d'un trait de plume les 300 praticiens qui en ont acquis la compétence depuis 1970 et exercent encore et les 1300 praticiens qui en ont acquis la qualification par le DESC depuis 1984.

Il est faux de dire que la formation à la réanimation est aujourd'hui commune entre nos deux disciplines. Des modules d'enseignement ont certes été organisés en commun dans l'interrégion Île-de-France, mais en aucun cas ces modules communs ne peuvent prétendre constituer une formation suffisante à la prise en charge de malades médicaux. L'objectif du DES dont nous demandons la création est également pédagogique. Nous voulons que les futurs réanimateurs soient mieux formés, que le cursus de formation leur permette d'acquérir au cours de l'internat une culture médicale approfondie et une expertise en réanimation. Nous voulons aussi pouvoir soutenir plus précocement leur orientation personnelle vers l'enseignement et la recherche.

Il est faux enfin et contradictoire encore de présenter la dimension européenne du common training framework comme la reconnaissance européenne exclusive d'un accès « multiculturel » à la réanimation interdisant d'en acquérir la qualification au cours d'une spécialité primaire. Le comité multidisciplinaire (Multiple Joint Committee of Intensive Care Medicine) et le board européen (European Board of Intensive Care Medicine) ont récemment défini ce socle commun de formation comme l'ensemble des connaissances, aptitudes et compétences nécessaires à l'exercice de la réanimation. Dans cette condition, la qualification peut être acquise en tant que spécialité primaire ou en tandem avec une autre spécialité primaire. Le message des instances européennes est simplement la possibilité laissée à chacun des pays d'organiser comme ils le veulent la formation des « intensivistes » à condition que cela respecte un socle commun de formation. Et la création d'un DES de réanimation n'est en rien contradictoire avec ce principe. Notre maquette de formation est assise sur le référentiel métier rédigé en 2011 à partir de la version française du référentiel européen
Cobatrice, expurgé des données strictement liées à l'anesthésie et aux situations périopératoires non compliquées.

Rappelons ici un petit point d'histoire. L'AR s'appelait chirurgicale jusqu'en 2004, date du passage de notre DESC de type 1 au DESC qualifiant. Le terme a disparu à la demande expresse de nos collègues et nous avons rayé du nôtre celui de médical. Comme les décrets de 2002 dont elle était la suite logique, cette disposition réglementaire en miroir concrétisait la possibilité d'un accès double à l'exercice professionnel de la réanimation, par là même véritablement forte et multiculturelle.

Nous pensons pour notre part que c'est le maintien d'une double formation par le DESAR et par le DES de réanimation qui permettra d'assurer la pérennité d'une véritable multidisciplinarité et la dimension « multiculturelle » dont parlent nos collègues. C'est ce qu'énoncent les décrets de 2002. C'est parce que nos services associent des médecins venus de ces deux cultures que nos patients sont bien et seront encore mieux soignés. Nous l'avons dit, répété, officiellement écrit.

Nous avons aussi tenté - hélas sans succès - de persuader nos collègues que l'AR ne prenait aucun risque à accepter cette évidence, parce qu'elle bénéficie d'une filiarisation indépendante et que nous garantissions comme aujourd'hui l'accès des internes du DESAR à nos services, sans lequel ils ne peuvent satisfaire à leur maquette de formation. Malheureusement, nous n'avons pas été entendus, et la position prise aujourd'hui par nos collègues d'AR menace clairement notre discipline.

Il n'y a rien d'illogique, de menaçant, d'insupportable à ce que nous demandions aujourd'hui la création d'un DES de réanimation, pour une meilleure formation de nos étudiants et pour mieux satisfaire le remplacement des praticiens que nous avons formés et qui vont nous quitter. Nous formons aujourd'hui 350 médecins au cours des trois années de cursus du DESC. Nous souhaitons basculer cette activité formatrice au cours de l'internat pour former mieux et former plus vite. Nous sommes de fait une spécialité exclusive reconnue, avec des centaines de praticiens, plus de 85 professeurs des universités-praticiens hospitaliers (PU-PH) et maîtres de conférence des universités-praticiens hospitaliers (MCU-PH), une sous-section du Conseil national des universités (CNU), un syndicat, des collèges, une société savante, qu'y a-t-il d'illégitime à proposer que la formation qui amène à notre spécialité se fasse aux mêmes moments et dans le même cadre que toutes les autres? Nous ne demandons rien d'autre que la même considération. Que l'on cesse de nous faire des procès d'intention machiavélique avant que trop de paroles, d'écrits, de manœuvres ou de menaces ne finissent par creuser un gouffre définitif entre nos deux disciplines.

Conflit d'intérêt : F. Fourrier déclare ne pas avoir de conflit d'intérêt. 